\title{
Complication rare mettant en jeu le pronostic vital lors de la pose d'implants symphysaires : cas clinique d'un hématome du plancher buccal
}

\author{
Marion Pesudo, Alexandre Alpy, Bruno Courtois, Arnaud L’Homme, Sarah Cousty \\ Service d'Odontologie, Département de Chirurgie orale, 3 chemin des Maraichers, \\ 31000 Toulouse, France \\ drmarionpesudo@voila.fr
}

Une patiente de 77 ans, traitée au long cours pour une hypertension artérielle (Cardensiel ${ }^{\circledR}$ ) et une hypothyroïdie (Levothyrox ${ }^{\circledR}$ ) est adressée aux urgences hospitalières. Suite à la pose de deux implants symphysaires pour stabiliser sa prothèse mandibulaire totale, et à la prise d'amoxicilline et de Lamaline $^{\circledR}$, elle présente plusieurs symptômes : dyspnée, œè̀me du plancher buccal et cervical, dysarthrie et paresthésies des membres supérieurs.

Les urgentistes diagnostiquent un œdème de Quincke lié à la prise médicamenteuse. Ils observent l'hématome du plancher buccal mais ils ne le considèrent pas comme seul responsable de la dyspnée. La patiente a reçu un traitement d'adrénaline par voie intraveineuse, du Solumédrol ${ }^{\circledR}$ et un antihistaminique. Après l'injection des amines vasopressives, l'électrocardiogramme objective une souffrance myocardique et l'augmentation des troponines ultrasensibles confirme le syndrome coronarien aigu. Les urgentistes instaurent alors un traitement par antiagrégant plaquettaire et anticoagulant, ainsi que de la clindamycine et du Solumédrol ${ }^{\circledR}$ pour éviter les complications infectieuses et diminuer l'œdème associé à l'hématome.

Le traitement local consiste à déposer les points de suture pour permettre l'écoulement du sang séquestré dans le plancher buccal et à surveiller. En cas d'apparition d'un nouveau saignement entrainant l'aggravation de la dyspnée ou d'une infection du caillot, il était envisagé d'effectuer le drainage au bloc opératoire sous anesthésie générale. L'évolution a été favorable sur le plan respiratoire, cardiaque et infectieux, et l'hématome a régressé spontanément.

Lors de la pose d'implant dans la symphysaire, l'effraction de la corticale pendant l'étape de forage peut être responsable de la formation d'un hématome dans le plancher buccal, sans que ce dernier soit favorisé par un traitement d'anticoagulant ou d'antiagrégant plaquettaire. Le pronostic vital peut être engagé lorsque l'hématome, à la fois sus et sous-mylohyoïdien se gagne les espaces latéro-pharyngés entrainant une détresse respiratoire aiguë, la compression de la langue et de l'épiglotte provoquant l'obstruction des voies aériennes supérieures.

Dans les premières heures post-opératoires, les symptômes de l'hémorragie du plancher buccal se manifestent par une tuméfaction endo et exo-buccal diffuse et indolore, entrainant une dyspnée, une dysphagie, une dysarthrie, une géne à la protraction et un refoulement de la langue induisant un risque d'asphyxie. Ces signes cliniques sont assimilables à une affection allergique de type adème de Quincke.

L'acte implantaire peut être à l'origine de complications hémorragiques graves surtout dans la région du cercle artériel péri-symphysaire. Malgré la survenue rare de cette complication potentiellement grave, la dépose des implants n'est pas indiquée. La gestion de cette complication vitale repose sur une approche pluridisciplinaire rapide et coordonnée. 